\title{
Theoretical Insights into MXene Termination and Surface Charge Regulation
}

\author{
Xuepeng Wang ${ }^{1}$, Gary M.C. Ong ${ }^{1}$, Michael Naguib² ${ }^{2}$, and Jianzhong $\mathrm{Wu}^{1, *}$ \\ ${ }^{1}$ Department of Chemical and Environmental Engineering, University of California, Riverside, CA, \\ 92521, United States \\ ${ }^{2}$ Department of Physics and Engineering Physics, Tulane University, New Orleans, LA 70118, \\ United States
}

\section{Supporting Information}

The support information provides additional details for calculating the Gibbs energy for formation for MXenes terminated with -O groups, the atomic configurations of different terminations, and additional results predicted from JDFT.

\section{The Gibbs energy of formation for MXenes with $O$ termination}

To identify the stable binding site for $\mathrm{O}$ termination, we calculated the Gibbs energy of formation according to

$$
\Delta_{f} G^{0}=G_{M O}^{0}-G_{M}^{0}-\mu_{O}
$$

where $G_{M O}^{0}$ and $G_{M}^{0}$ are the Gibbs free energy of MXene with O termination and bare Mxene at standard conditions, i.e., room temperature and pressure $p=1 \mathrm{~atm}$ in an aqueous solution. The Gibbs free energy consists of the total energy (E) with solvation effect and vibration free energy including zero-point energy (ZPE) and vibration entropy

$$
G^{0}=E+\mathrm{ZPE}+T S_{v i b}
$$

The chemical potential of $\mathrm{O}$ is selected according to the Gibbs free energy of oxygen gas at the standard condition 


$$
\begin{aligned}
\mu_{O} & =\frac{1}{2} G_{O_{2}}^{0} \\
& =\frac{1}{2}\left(E_{O_{2}}+\mathrm{ZPE}_{\mathrm{O}_{2}}+\frac{7}{2} k_{B} T-T S_{O_{2}}\right)
\end{aligned}
$$

JDFT calculations yield the total energy with solvation effect by the implicit solvent model. The ZPE and vibration entropy at room temperature are evaluated in vacuum.

\section{Configurations of mixing $\mathrm{O}$ and $\mathrm{OH}$ terminations}

Since there are 9 binding sites for $\mathrm{O}$ and $\mathrm{OH}$ in a $3 \times 3 \times 1 \mathrm{Ti}_{3} \mathrm{CN}$ supercell, when the amount of $\mathrm{OH}$ termination is more than 1 but less than 8 , the different arrangement pattern of $\mathrm{O}$ and $\mathrm{OH}$ terminations will lead to different configurations as displayed in Figure S1. The configurations is named as "Hni", where $n$ is the amount of $\mathrm{OH}$ terminations, and $i=\mathrm{a}, \mathrm{b}, \mathrm{c}$, or $\mathrm{d}$ stands for the different configuration for the same $n$. For the $\mathrm{O}$ and $\mathrm{OH}$ in a $3 \times 3 \times 1 \quad \mathrm{~V}_{2} \mathrm{C}$ supercell, the configurations are the same with those in a $3 \times 3 \times 1 \mathrm{Ti}_{3} \mathrm{CN}$ supercell since they have the same amount of binding site and hexagonal lattice. While, the energy change caused by the different configurations is relatively small as shown in Table S1 and S2. Table S1 and S2 also reveal repulsion interactions between the $\mathrm{OH}$ terminations. Because the lowest energy structure always has $\mathrm{OH}$ terminations and stays away from each other as much as possible, especially for the $\mathrm{H} 2$, $\mathrm{H} 3$ and H4. For example, $\mathrm{H} 3 \mathrm{a}$ has the lowest energy as there are no other $\mathrm{OH}$ around each $\mathrm{OH}$. 


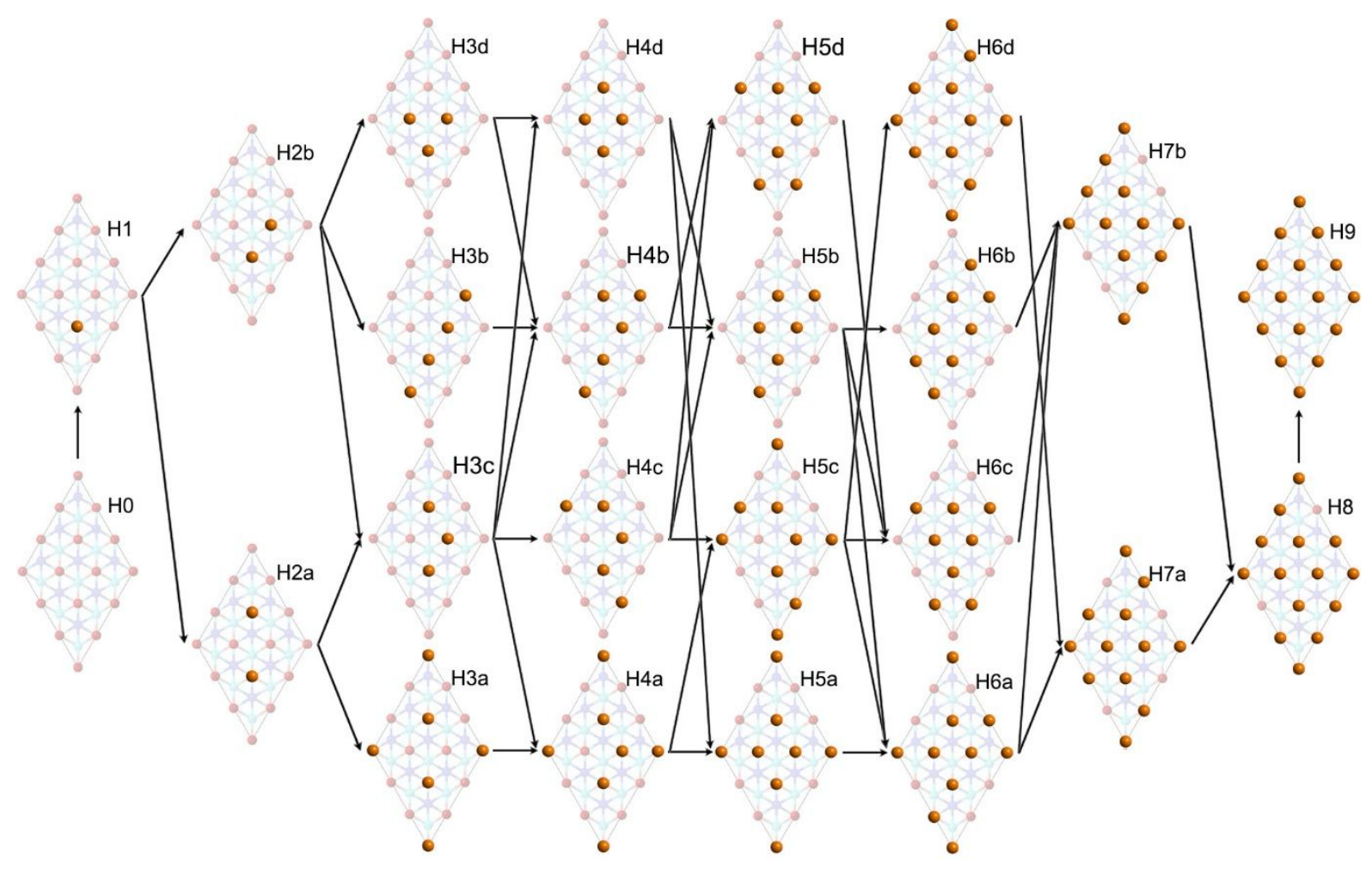

Figure S1. Possible surface configurations for different amount of proton coverage in a $3 \times 3 \times 1$ $\mathrm{Ti}_{3} \mathrm{CN}$ supercell. The position of $\mathrm{OH}$ terminations are highlighted by large orange balls. The black arrows indicate possible structural transitions during protonation reactions.

Table S1 Relative energy (eV) of configurations for different amount of proton coverage in a $3 \times 3 \times 1 \mathrm{Ti}_{3} \mathrm{CN}$ supercell. The lowest energy of configurations for each proton coverage is set to be zero.

\begin{tabular}{ccccccc}
\hline & H2 & H3 & H4 & H5 & H6 & H7 \\
\hline a & $\mathbf{0 . 0 0 0}$ & $\mathbf{0 . 0 0 0}$ & $\mathbf{0 . 0 0 0}$ & 0.011 & 0.016 & 0.042 \\
b & 0.063 & 0.136 & 0.038 & 0.010 & 0.0001 & $\mathbf{0 . 0 0 0}$ \\
c & -- & 0.086 & 0.009 & 0.021 & $\mathbf{0 . 0 0 0}$ & -- \\
d & -- & 0.141 & 0.048 & $\mathbf{0 . 0 0 0}$ & 0.092 & -- \\
\hline
\end{tabular}


Table S2 Relative energy (eV) of configurations for different amount of proton coverage in a $3 \times 3 \times 1 \quad \mathrm{~V}_{2} \mathrm{C}$ supercell

\begin{tabular}{ccccccc}
\hline & H2 & H3 & H4 & H5 & H6 & H7 \\
\hline a & $\mathbf{0 . 0 0 0}$ & $\mathbf{0 . 0 0 0}$ & $\mathbf{0 . 0 0 0}$ & 0.017 & 0.030 & $\mathbf{0 . 0 0 0}$ \\
b & 0.109 & 0.227 & 0.066 & $\mathbf{0 . 0 0 0}$ & $\mathbf{0 . 0 0 0}$ & 0.003 \\
c & -- & 0.138 & 0.034 & 0.015 & 0.021 & - \\
d & -- & 0.223 & 0.057 & 0.009 & 0.161 & -- \\
\hline
\end{tabular}

\section{Effect of the supercell size}

To investigate how the size of supercell affect the Gibbs energy per site at point of zero charge (PZC), we perform the calculation in a $4 \times 4 \times 1 \mathrm{~V}_{2} \mathrm{C}$ supercell. There are 16 binding sites in a $4 \times 4 \times 1$ supercell. When the $\mathrm{H}$ coverage $\theta$ is 0 or 1 , the configurations should be the same for a $3 \times 3 \times 1$ and a $4 \times 4 \times 1$ supercell due to the periodic boundary conditions. As expected, the Gibbs energy per site are the same for a $3 \times 3 \times 1$ and a $4 \times 4 \times 1$ supercell when $\theta=0$ and $\theta=1$. See Figure S2. Moreover, the Gibbs energy per site at PZC vs. $\theta$ (for $\theta<0.2$ ) calculated by a $4 \times 4 \times 1$ supercell could match well with that given by a $3 \times 3 \times 1$ one. Here, for the $4 \times 4 \times 1$ supercell we only calculated the Gibbs energy in the range of $\theta<0.2$. Because as $\theta$ increase, the number of configurations will increase dramatically since additional binding sites lead to more complex arrangement pattern of $\mathrm{O}$ and $\mathrm{OH}$ terminations. While, the results above could verify that the size of supercell used will not affect the Gibbs energy per site at PZC as a function of $\theta$. 


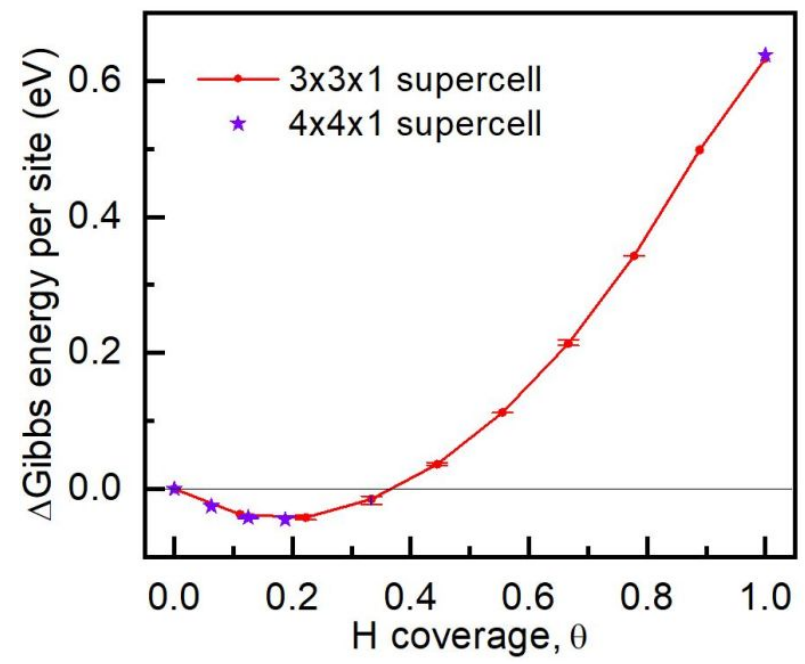

Figure S2. Gibbs energy per site at PZC vs. $\mathrm{H}$ coverage in $3 \times 3 \times 1$ (red) and $4 \times 4 \times 1$ (purple) $\mathrm{V}_{2} \mathrm{C}$ supercells. The error bars stand for the difference due to different configurations.

\section{DOS of $\mathrm{V}_{2} \mathrm{C}$}

Similar with $\mathrm{Ti}_{3} \mathrm{CN}$, the total density of states (DOS) for $\mathrm{H} 0, \mathrm{H} 2 \mathrm{a}$, and $\mathrm{H} 9$ configurations shown in Figure S3a exhibit the properties of a conductor without band gap. As discussed in the main text, due to the unsaturated dangling bond, $\mathrm{O}$ in $\mathrm{H} 2 \mathrm{a}$ has more states in the valance band which mainly comes from the O termination. See Figure S3b. Also, according to Figure S3c, the contribution of $\mathrm{H}$ is much smaller as reflected by the quite few states around $\mathrm{E}_{\mathrm{f}}$ until the surface is fully covered by $\mathrm{OH}$. 


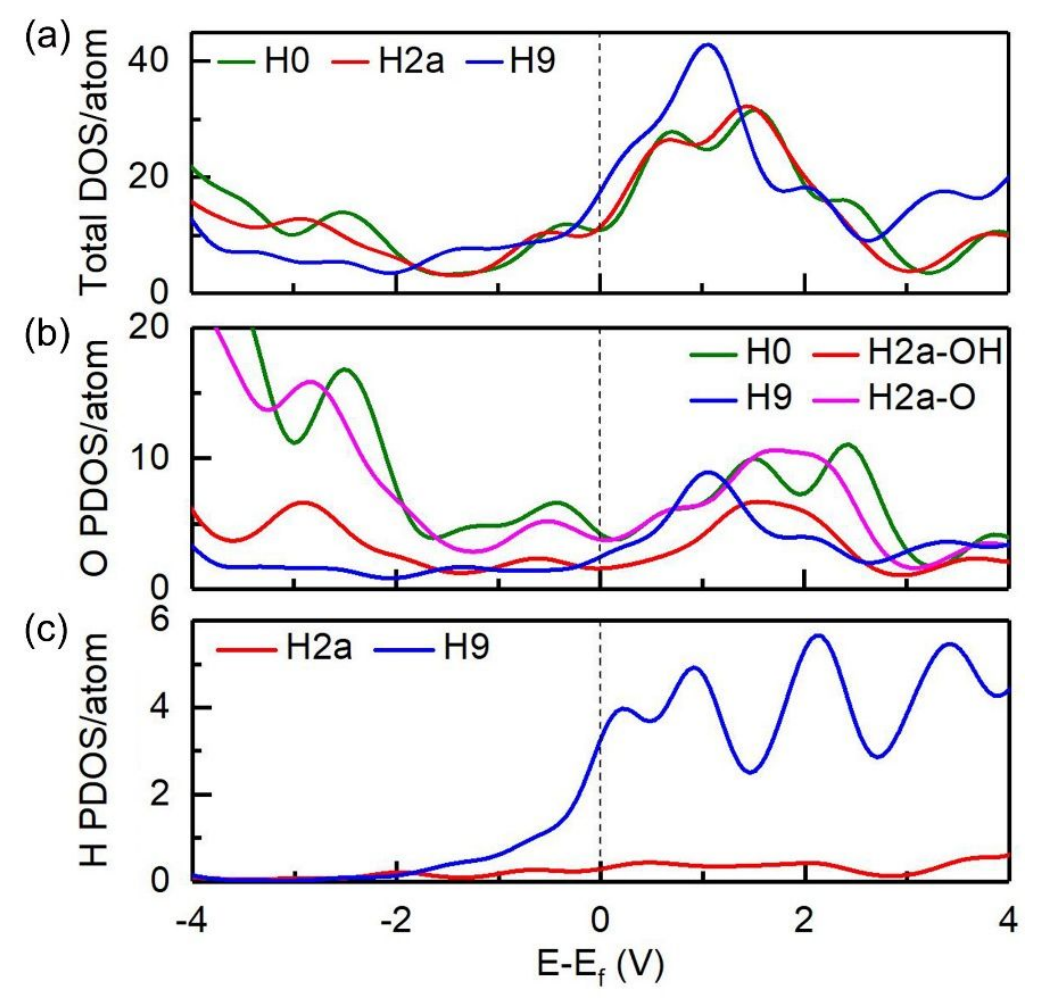

Figure S3. Comparisons of (a) the total DOS, PDOS of (b) O and (c) $\mathrm{H}$ for neutral H2a and H9 (fully terminated by $\mathrm{OH}$ ) $\mathrm{V}_{2} \mathrm{C}$ MXene. For the PDOS of $\mathrm{O}, \mathrm{H} 2 \mathrm{a}-\mathrm{OH}$ and $\mathrm{H} 2 \mathrm{a}-\mathrm{O}$ stands for the surface $\mathrm{O}$ atoms that binding with a $\mathrm{H}$ and without $\mathrm{H}$ binding, respectively.

\section{Net charge distribution}

The net charge distribution is reflected by the charge (or electronic) density difference (CDD) iso-surface. As shown in Figure S4, we select three different iso-surfaces to display the net charge distribution. From the three iso-surfaces of CDD, we could obtain the same conclusions: the positive net charge is mainly distributed around $\mathrm{O}$ while the negative net charge is evenly distributed on the surface. Therefore, the net charge distribution characteristics do not depend on the selection of the iso-surface. In addition, $\mathrm{V}_{2} \mathrm{C}$ also holds the similar uniform net charge distribution feature with $\mathrm{Ti}_{3} \mathrm{CN}$ according to the $\mathrm{CDD}$ shown in Figure S5. 
(a) $\mathrm{H}_{2} \mathrm{a}^{-}$
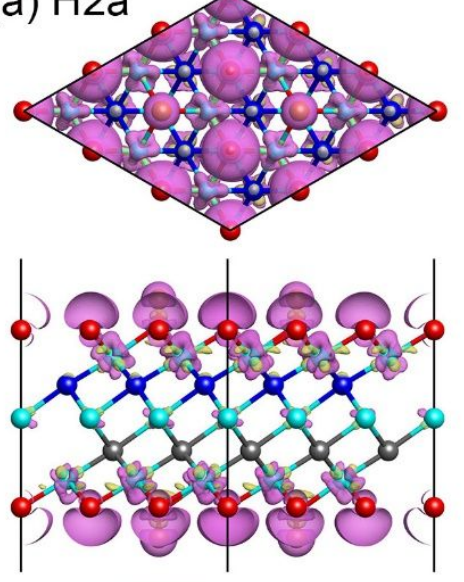

0.001 a.u.

(b) $\mathrm{H}_{4} \mathrm{a}^{+}$
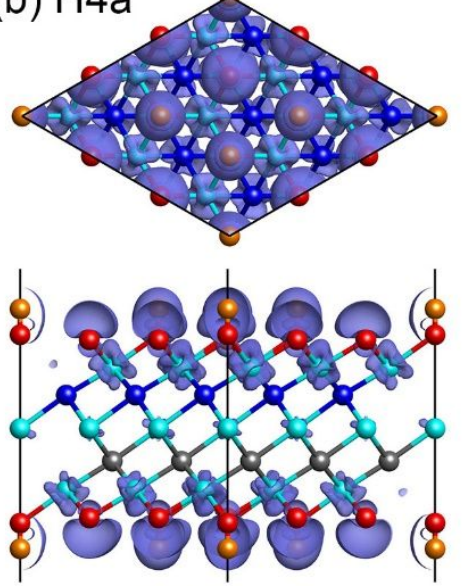

-0.001 a.u.
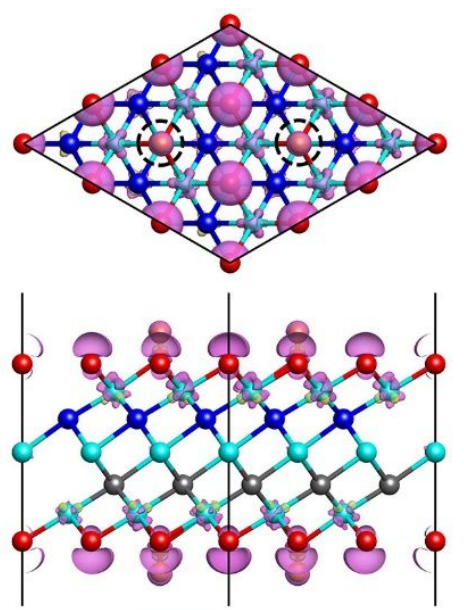

0.002 a.u.
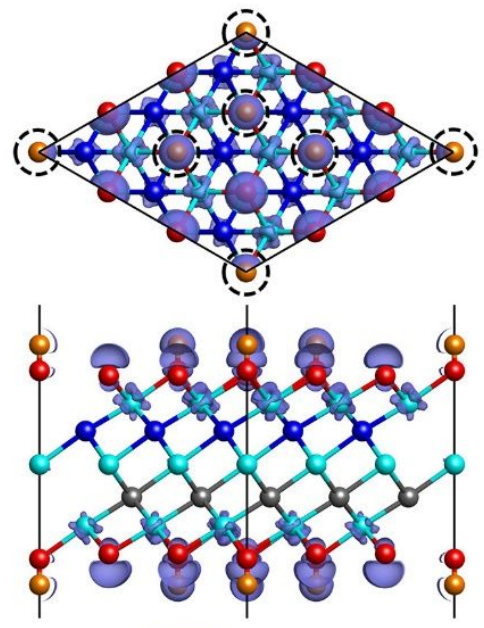

-0.002 a.u.
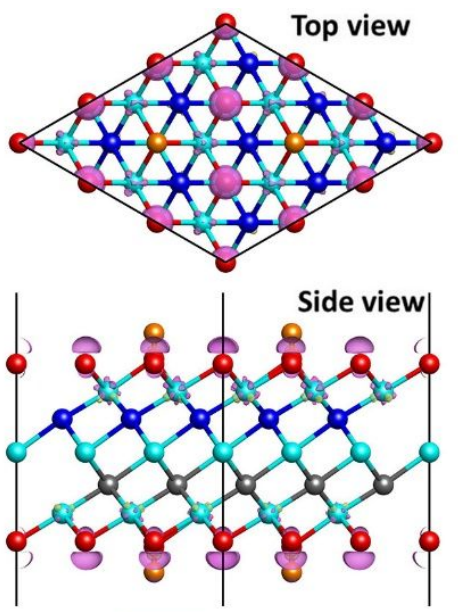

0.003 a.u.
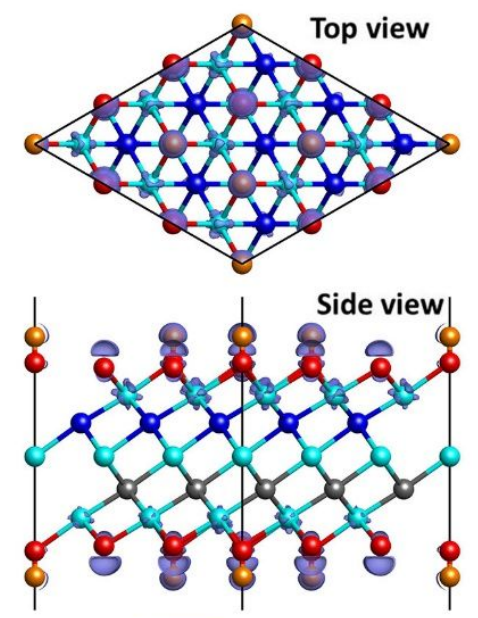

-0.003 a.u.

Figure S4. Charge density difference (CDD) with different iso-surface for (a) negatively and (b) positively charged $\mathrm{Ti}_{3} \mathrm{CN} .1$ a.u. $=1 \mathrm{e}^{-} / \mathrm{a}_{0}{ }^{3}$. The position of $\mathrm{H}$ atoms is highlighted by black dash circles on the top view of structure. 

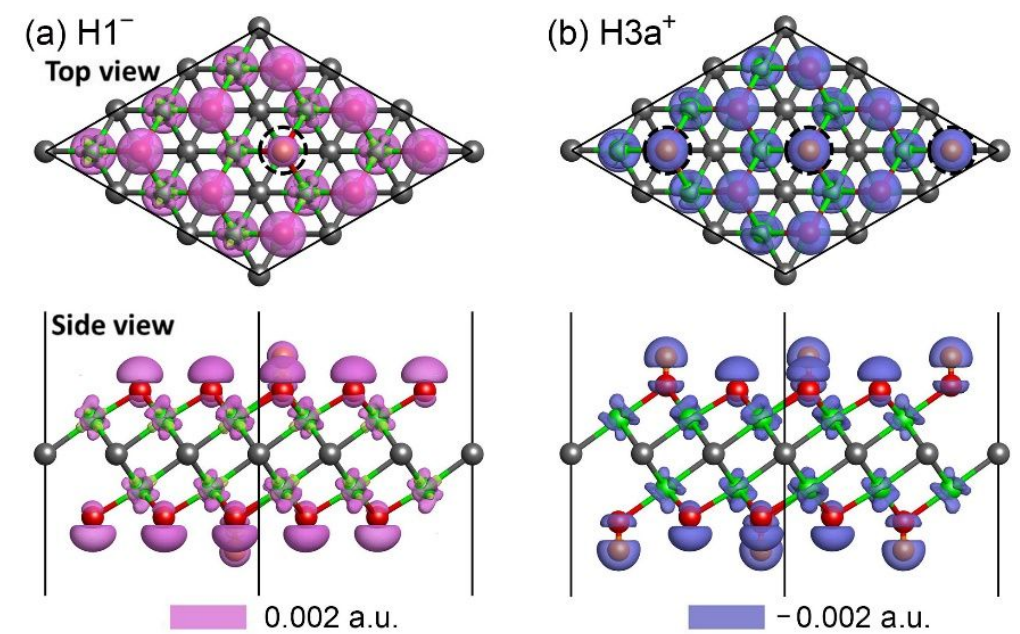

Figure S5. Distribution of the net charge on $\mathrm{V}_{2} \mathrm{C}$ MXene as reflected by the iso-surface of CDD. The position of $\mathrm{H}$ atoms is highlighted by black dash circles on the top view of structure. 